\section{Computer dissection of the auditory evoked potential: A comment on Schweitzer}

\author{
STEVEN A. HILLYARD \\ Department of Neurosciences \\ University of California, San Diego \\ La Jolla, California
}

Schweitzer (1977) has recently reported that the auditory evoked potential (AEP) to a short-duration tone burst can be dissected into separate "ON" and "OFF" components, evoked by the rising and falling edges of the stimulus, respectively. The experimental method involved the collection of natural AEPs or "multiple responses" (MRs) to short tones (25 and $75 \mathrm{msec}$ in duration) as well as the separate $\mathrm{ON}$ and OFF responses to a 2,000-msec tone. On the assumption that the natural AEP to the 25-msec tone (25-MR) is composed of overlapping, separable ON and OFF components, Schweitzer reasoned that subtraction of the 2,000-ON response from the 25-MR would yield a "dissected" component (termed d25-OFF) which represents the AEP to the offset of the $25-\mathrm{msec}$ tone. Similarly, subtraction of the 2,000-OFF response from the natural 25-MR (at the appropriate delay interval) should yield a residual AEP which represents the activity evoked by the onset of the 25-msec tone (termed the d25-ON). Similar computer dissections were performed upon the AEP to the 75-msec tone. Employing this additive logic further, computer-synthesized AEPs to the shorter tones were constructed by adding together the natural 2,000-ON and 2,000-OFF AEPs, offset by the appropriate intervals.

Schweitzer reported that the computer-dissected ON-responses (d25-ON, d75-ON) were "remarkably similar" to the natural 2,000-ON response in both waveshape and amplitude, while the dissected OFF responses (d25-OFF, d75-OFF) were reported to be "obviously similar" to the natural 2,000-OFF response. Furthermore, there were "additional similarities" noted between the computer-synthesized s25-MR and s75-MR responses and the naturally occurring 25-MR and 75-MR AEPs. Based on these apparent similarities of waveshape and amplitude between the naturally occurring responses and their computer-dissected or synthesized counterparts, Schweitzer reached the following set of interrelated conclusions:

(1) "The observed [AEP] to short duration stimuli probably is the result of overlapping responses to the onset and offset of stimulation."

This work was supported in part by NIMH Grant MH 25594-04. I thank T. W. Pjeton, S. Van Voorhis, and R. Galambos for valuable discussions.
(2) The AEP to a short tone "appears to be the result of temporal summation of separate responses to stimulus onset and offset, rather than a direct neural interaction."

(3) "It seems quite unlikely that the ON and OFF responses are mediated by a single physiological mechanism sensitive to changes occurring over such short durations."

(4) "The data suggest that $\mathrm{ON}$ and OFF responses are mediated by independent physiological mechanisms."

Schweitzer's report presents one of those remarkable occasions where the same set of data may be subjected to precisely opposite interpretations, depending upon which assumptions and premises are taken into consideration. In particular, the components in the dissected OFF waveform reported by Schweitzer may be interpreted, not as offset-evoked responses, but as derivatives of certain other properties of the human AEP which were confounded in these waveform manipulations. These properties include interactions between $\mathrm{ON}$ and OFF responses to successive tones due to their refractory periods (Pfefferbaum, Buchsbaum, \& Gips, 1971) and the slow, auditory-evoked sustained potentials (Keidel, 1976), which overlap both the ON and OFF responses to long-duration tones. Taking these factors into account, it is possible to arrive at quite a contrary set of conclusions, namely that: (1) there are very strong interactions between closely succeeding ON and OFF responses; (2) these interactions are so strong that the OFF component is of negligible amplitude for tones as short as $25-75 \mathrm{msec}$; (3) hence, the AEP to a short-duration tone consists almost exclusively of an $\mathrm{ON}$ response to the beginning of the tone.

As detailed below, it would appear that the findings of Schweitzer and of other investigators (Pfefferbaum et al., 1971; Hillyard \& Picton, Note 1) are more consistent with this second set of conclusions.

The first indication that Schweiizer's "dissected" OFF responses may not contain appreciable components elicited by stimulus offset comes from examining the AEP latencies in Figure 2. It is seen that the latency of the major vertex-positive deflection (wave " $D$ ') is virtually identical in the d25-OFF and d75-OFF waveforms at most intensities. If these dissected AEPs actually represent offset-evoked components, as Schweitzer proposes, it would seem that the latency of component $\mathrm{D}$ should be delayed by some $50 \mathrm{msec}$ in the d75-OFF waveform relative to the d25-OFF. The absence of such a delay suggests that the dissected wave $\mathrm{D}$ actually derives from components that are time locked to stimulus onset 
in the natural AEPs and are subtracted from one another to form the dissected AEP.

The appearance of onset-locked components in Schweitzer's dissected OFF waveforms may be accounted for by considering the long refractory period of the late components of the AEP (Picton, Hillyard, \& Galambos, 1976). A strong refractory interaction between the OFF AEP to a long-duration tone and the ON AEP to the succeeding tone in a series was revealed clearly in the report by Pfefferbaum et al. (1971). These investigators recorded vertex AEPs to tone bursts presented once every $3,000 \mathrm{msec}$, with tone durations of either 500 or $2,500 \mathrm{msec}$. They found that the AEP to tone onset was markedly attenuated (by some $48 \%$ ) when the preceding tone offset occurred $500 \mathrm{msec}$ earlier rather than $2,500 \mathrm{msec}$ earlier. That is, the longer the silent interval before a tone onset, the larger was its AEP measured as the peak-to-peak amplitude between the $N_{1}(90-130 \mathrm{msec})$ and $P_{2}(170-200 \mathrm{msec})$ components. The $\mathrm{N}_{1}$ and $\mathrm{P}_{2}$ waves are the most prominent components in the AEP at the vertex, and are labeled by Schweitzer as $A, E$, or $C$ (for $N_{1}$ ) and $B, D$, or $F$ (for $P_{2}$ ), depending on the stimulus condition. These straightforward findings of Pfefferbaum et al. are, in themselves, very difficult to reconcile with the hypothesis that $O N$ and OFF AEPs are mediated by independent (i.e., noninteracting) mechanisms.

The presence of strong interactions between successive OFF and $O N$ responses would invalidate one of the major assumptions behind the additive logic on which Schweitzer's waveform manipulations are based. Specifically, the natural ON AEP to the 2,000-msec tone cannot be considered as equivalent to the ON portion of the $25 \mathrm{MR}$, because the latter was evoked after a 3,975-msec silent interval (Schweitzer presented tones once every $4 \mathrm{sec}$ ), while the former was elicited after only $2,000 \mathrm{msec}$ of silence. Accordingly, it might be expected that the $25 \mathrm{MR}$ and $75 \mathrm{MR}$ AEPs would be larger than the 2,000-ON AEP, not because the former contained additional OFF components, but because they were composed of less refractory ON responses. From these considerations, subtracting the 2,000-ON AEP from the 25-MR would not yield a dissected OFF response, but rather a wave that is the difference between a large-amplitude ON response ( $25 \mathrm{MR})$ and a smaller-amplitude $\mathrm{ON}$ response $(2,000-\mathrm{ON})$. Thus, it seems possible that the d25-OFF waveform represents the difference between two ON AEPs in different states of neural recovery rather than a "dissected" OFF response.

In support of this interpretation, Hillyard and Picton (Note 1) have recently replicated Schweitzer's procedures using AEPs recorded both at 4- and 10-sec ISIs. At the 4-sec ISI, the $25 \mathrm{MR}$ contained a larger $\mathrm{N}_{1}-\mathrm{P}_{2}$ response than the $2,000-\mathrm{ON} \mathrm{AEP}$, but at the 10-sec ISI, this difference disappeared in some subjects and was reversed in others. Accordingly, an appreciable $\mathrm{N}_{1}-\mathrm{P}_{2}$ was evident in the d-25 OFF waveform only at the 4-sec ISI. This result is in accord with the hypothesis of strong refractory interactions between successive ON and OFF responses and is inconsistent with the proposal that the $25 \mathrm{MR}$ contains a substantial OFF-evoked component.

In these experiments, the d25-OFF waveform may resemble the 2,000-OFF AEP at the 4-sec ISI because of the diphasic, $\mathrm{N}_{1}-\mathrm{P}_{2}$ morphology that characterizes both ON and OFF responses (e.g., Clynes, 1969; Keidel, 1976; Onishi \& Davis, 1968; Spychala, Rose \& Grier, 1969). Hence, the subtraction of one ON response from another, as well as the addition or subtraction of OFF and $\mathrm{ON}$ responses will, at small phase lags, result in a wave that retains the general $\mathrm{N}_{1}-\mathrm{P}_{2}$ waveshape. Similarities of waveform cannot, therefore, be used as unequivocal criteria of whether a dissected component is equivalent to a particular type of natural AEP. Schweitzer also used amplitude similarities as a criterion for identifying certain dissected or synthesized AEPs with natural AEPs. It appears from her graphs, however, that there are substantial discrepancies in amplitude between waves that ought to be identical under the hypothesis of additive, noninteractive $\mathrm{ON}$ and OFF components. For example:

(1) The 2,000-OFF AEP ( $\mathrm{N}_{1}-\mathrm{P}_{2}$ amplitude) was about $45 \%$ larger than the d25-OFF response (Figure 5 regression line at $86 \mathrm{~dB}$; also Figure 8), although the two should be identical if noninteracting $O N$ and OFF responses can be dissected out. This discrepancy can easily be accounted for by assuming that the relative amplitudes of the 2,000-ON and 25-MR AEPs, which are subtracted to form the d25-OFF AEP, are determined primarily by the particular ISI that is chosen. If a longer ISI had been used which minimized the effects of refractory interactions, then the d25-OFF wave would be even smaller in relation to the 2,000-OFF response (Hillyard \& Picton, Note 1 ).

(2) The d75-ON response was about $38 \%$ larger than the d25-ON response (Figure 6, regression lines at $86 \mathrm{~dB}$ ), although they should be equal under the hypothesis of independent ON and OFF responses. This discrepancy can be accounted for by considering that the $\mathrm{d} 25-\mathrm{ON}$ response was formed by subtracting the 2,000-OFF response from the $25 \mathrm{MR}$ with $\mathrm{N}_{1}-\mathrm{P}_{2}$ waves of the two AEPs nearly in phase (offset by $35 \mathrm{msec}$ ), thus resulting in a small difference wave. To form the $d 75-O N$ response, however, the 2,000-OFF AEP was subtracted from the $75 \mathrm{MR}$ at a larger phase lag $(85 \mathrm{msec})$, such that the latter wave was scarcely attenuated by the subtraction. This pattern of AEP amplitudes (Figure 6) is in accord with the basic proposition that the 25 and $75 \mathrm{MRs}$ (and not the $\mathrm{d} 25-\mathrm{ON}$ waves) represent rather pure, 
and equivalently large, $\mathrm{ON}$ responses. If, as Schweitzer claims, "the OFF response appears to contribute more to the response evoked by the shorter duration stimulus of $25 \mathrm{msec}$ than the longer duration stimulus of $75 \mathrm{msec}$," why is it that the 25 and 75 MRs were so nearly identical in amplitude throughout the entire range of stimulus intensities (Figure 6)?

(3) A similar line of reasoning can explain why the synthetic s25-MR turned out to be substantially larger than its natural counterpart (25-MR), while the s75-MR was substantially smaller than the 75-MR (Figure 7). To form the s25-MR, the $\mathrm{N}_{1}-\mathrm{P}_{2}$ waves in the 2,000-ON and 2,000-OFF responses were added nearly in phase, while for the s75-MR they were added mostly out of phase. In neither case were the synthesized wave amplitudes close to what they should have been under the hypothesis of separate, additive $O N$ and OFF responses. Given these wide amplitude discrepancies, it is difficult to accept Schweitzer's statement (p. 92) that "further indication of the existence of the OFF response at short durations can be seen in the synthesized multiple response waveforms."

Certain distortions can be seen in Schweitzer's synthesized and dissected waveforms which are also consistent with the thesis that the 25 and 75 MRs did not contain appreciable OFF components. For example, the s75 MR contained a large, vertex-positive bulge in the 200- to 300-msec range (attributable to the delayed addition of the $\mathrm{P}_{2}$ wave of the 2,000-OFF response), while the $75 \mathrm{MR}$ lacked this feature. Similarly, the late, vertex-negative bulge in the d75-ON response was lacking in the purportedly equivalent $2,000-\mathrm{ON}$ response. These distortions most likely arose from the inappropriate addition and subtraction, respectively of an OFF response that was not present in the natural waveforms.

Schweitzer seemed to recognize some of these difficulties in the discussion on pp. 92-93 and suggested that "the amplitude of s25 MR may be artificially inflated because the ON and OFF [AEPs] summed together to produce the synthesized response may not be (and probably are not) identical to the ON and OFF responses to the shorter 25-msec-duration stimulus." Evidently, she did not consider these discrepancies between the natural and synthetic AEPs to be very serious, for she went on to suggest that only "a small amount of the total variance may be due to neural interaction." It seems clear, however, that these discrepancies were substantial and in accordance with the hypothesis of strong neural interactions.

Further evidence against the existence of an appreciable OFF response in the AEP to short-duration tones comes again from the experiment of Pfefferbaum et al. (1971). They found that shortening tone duration from 2,500 to $500 \mathrm{msec}$ resulted in a marked decline in the OFF $N_{1}-P_{2}$ (by 57\%). A systematic decline in the amplitude of the OFF response for shorter tones was also reported by Hillyard and Picton (Note 1) and by Picton, Woods, and Proulx (Note 2). If one extrapolates this trend, it is clear that the OFF response to a tone of $25-75 \mathrm{msec}$ would be very small indeed and in no way comparable to the robust OFF responses which occur to tones of 2,000 $2,500 \mathrm{msec}$. It would seem that these results can only be interpreted as indicative of strong neural interactions between $\mathrm{ON}$ and $\mathrm{OFF}$ responses.

Employment of dc recording techniques reveals another feature of these AEPs which confounds the subtraction procedure used by Schweitzer. Throughout the longer-duration tones is a negative potential shift, the well-known auditory-evoked sustained potential (Keidel, 1976; Köhler \& Wegener, 1955; Picton, Hillyard, \& Galambos, 1976). The sustained potential (SP) may begin as early as $150 \mathrm{msec}$ after tone onset, and thus may overlap the $P_{2}$ component elicited by the 2,000-msec tone (Picton, Woods, \& Proulx, Note 2). Accordingly, subtraction of a 2,000-ON response which includes this negativity from the 25 MR which does not will result in a sustained positivity in the d25-OFF waveform, beginning in the $\mathrm{P}_{2}$ latency range. With the ac amplification used by Schweitzer (T.C. $=.45 \mathrm{sec}$ ), such a sustained positivity would be converted to a broad phasic wave that resembles a $P_{2}$ component. This positive wave would be attributable, not to an OFFcomponent in the $25 \mathrm{MR}$ but to the negative SP in the 2,000-ON response. Given the likelihood of overlap between the SP and the $P_{2}$ wave in the $2,000-O N$ response, it is difficult to rule out the SP as a contributor to component $D$ the $\mathrm{d} 25$-OFF response.

Since the negative SP terminates abruptly at the offset of long-duration tones, the resulting positive shift also summates or interacts with whatever $\mathbf{N}_{1}-\mathbf{P}_{2}$ components are elicited by tone offset (Hillyard \& Picton, Note 1). The result is an artificial inflation of the $N_{1}-P_{2}$ measure of the 2,000-OFF response by the positive-going dc shift, which is converted to a broad positive wave by an ac amplifier. Such a slow positivity is evident in the 2,000-OFF tracings of Schweitzer's Figure 2. The presence of this positive $\mathrm{dc}$ offset is yet another factor which makes it difficult to compare the OFF responses to long tones with the hypothetical OFF response to short tones. In fact, the apparent similarity between the d25-OFF and 2,000 -OFF waveforms could arise in part from the presence of an inverted SP onset in the former and a natural SP offset in the latter, rather than from any offset-evoked component that is common to both long and short tones.

A final factor which could differentially influence the AEPs to long and short tones is the temporal integration of stimulus energy, which is known to affect loudness as well as the AEP (see Keidel, 1976 
and Onishi \& Davis, 1968, for reviews). Depending on stimulus intensity, loudness may increase systematically as a function of tone duration well beyond $25 \mathrm{msec}$. Thus, any residual difference wave between the $25 \mathrm{MR}$ and 2,000-ON AEPs which cannot be accounted for by refractory interactions or by summation with the SP could reflect temporal integration within the ON response rather than the presence of an OFF response.

Because of these confounding factors, the data analysis presented by Schweitzer does not conclusively demonstrate the existence of an appreciable OFF component in the AEP to short tones. These data, as well as those from previous studies, can be accounted for more fully and accurately by the proposition that $\mathrm{ON}$ and OFF responses interact strongly, such that the $N_{1} P_{2}$ components of the AEP to a brief tone consist almost exclusively of an ON response. This conclusion is consonant with proposals that auditory-evoked ON and OFF responses in the late cortical AEP originate from closely related or identical neural systems (Clynes, 1969; McCandless \& Rose, 1970).

\section{REFERENCE NOTES}

1. Hillyard, S. A., \& Picton, T. W. ON and OFF components in the auditory ewoked potential. Submitted for publication.

2. Picton, T. W., Woods, D. L., \& Proulx, G. B. Human auditory sustained potentials. II: Stimulus relationships. Submitted for publication.

\section{REFERENCES}

Clynes, M. Dynamics of vertex evoked potentials: The R-M brain function. In E. Donchin \& D. B. Lindsley (Eds.), Average evoked potentials-Methods, results, and evaluations. Washington, D.C: NASA, 1969. Pp. 363-374.

KEIDEL, W. The physiological background of electric response audiometry. In W. D. Keidel \& W. D. Neff (Eds.), Handbook of sensory physiology (Vol. V/3). Auditory system. Clinical and special topics. Berlin: Springer-Verlag, 1976. Pp. 105-231.

KöHLER, W., \& Wegener, J. Currents of the human auditory cortex. Journal of Cellular and Comparative Physiology, 1955, 45, 25-54.

McCandless, G. A., \& Rose, D. E. Evoked cortical responses to stimulus change. Journal of Speech and Hearing Research, 1970, 13, 624-634.

OnishI, S., \& Davis, H. Effects of duration and rise time of tone bursts on evoked potentials. Journal of the Acoustical Society of America, 1968, 44, 582-591.

Pfefferbaym, A., Buchs baum, M., \& Gips, J. Enhancement of the average evoked response to tone onset and cessation. Psychophysiology, 1971, 8, 332-339.

Picton, T. W., Hillyard, S. A., \& Galambos, R. Habituation and attention in the auditory system. In W. D. Keidel \& W. D. Neff (Eds.), Handbook of sensory physiology (Vol. V). Berlin: Springer-Verlag, 1976. Pp. 343-387.

SCHWEITZER, P. K. Auditory evoked brain responses: Comparison of ON and OFF responses at long and short durations. Perception \& Psychophysics, 1977, 22, 87-94.

Spychala, P., Rose, D., \& Grier, J. Comparison of the "ON" and "OFF" characteristics of the acoustically evoked response. International Audiology, 1969, 8, 416-417.

(Received for publication March 6, 1978; accepted March 21, 1978.) 